\title{
The Application of Microdosimetric Principles to Radiation Hardness Testing
}

\author{
Jeremy D. Northum and Stephen B. Guetersloh \\ Department of Nuclear Engineering, Texas AઐM University, MS 3133, College Station, TX 77840, USA \\ Correspondence should be addressed to Jeremy D. Northum; jnorthum@tamu.edu
}

Received 10 October 2013; Accepted 22 November 2013; Published 12 January 2014

Academic Editor: Jakrapong Kaewkhao

Copyright ( 2014 J. D. Northum and S. B. Guetersloh. This is an open access article distributed under the Creative Commons Attribution License, which permits unrestricted use, distribution, and reproduction in any medium, provided the original work is properly cited.

\begin{abstract}
Chord length distributions for rectangular parallelepipeds of various relative dimensions were studied in relation to radiation hardness testing. For each geometry, a differential chord length distribution was generated using a Monte Carlo method to simulate exposure to an isotropic radiation source. The frequency and dose distributions of chord length crossings for each geometry, as well as the means of these distributions, are presented. In every case, the dose mean chord length was greater than the frequency mean chord length with a $34.5 \%$ increase found for the least extreme case of a cube. This large increase of the dose mean chord length relative to the frequency mean chord length demonstrates the need to consider rare, long-chord-length crossings in radiation hardness testing of electronic components.
\end{abstract}

\section{Introduction}

Microdosimetry is the study of the patterns of energy deposition from ionizing radiation interacting with microscopic volumes much smaller than the range of secondary particles generated by the incident particle. Since its inception less than 50 years ago, it has been very important in the field of radiobiology [1]. Because of this, metrics evolved around a standard geometry of a $1 \mu \mathrm{m}$ sphere [2] and published results from experimental measurements are related to radiation interaction with a spherical or cylindrical detector. Over the past decade or so, increasingly small components have been developed to meet the demands of the electronics industry and, as a result, the amount of charge necessary for inducing a single event effect (SEE) becomes correspondingly smaller [3]. In deep space SEE production results mainly from the traversal by heavy ions that make up the galactic cosmic ray (GCR) spectrum. In low-Earth orbits SEE production is dominated by proton-induced spallation reactions [3]. As many of the components manufactured today can easily have dimensions on the order of several micrometers, the principles of microdosimetry have application in stability testing of these microchips intended for use in radiation fields. However, the differences in geometry between the sphere and the parallelepiped need to be considered.

A significant amount of work has been performed at accelerator facilities to test the radiation hardness of electronic components. Attention is often given to the influence of geometric factors on SEE rates. While several recent studies have considered the incident angle of the beam in radiation hardness testing [4-7], many apparently do not $[8,9]$ and this is a crucial factor when analyzing upsets as a function of energy delivered. It is recognized that some radiation hardness testing has been focused on the influence of beam energy, linear energy transfer (LET), and other nongeometry factors or has had geometry factors as a secondary area of study.

Even with that recognition in mind, it seems as though insufficient consideration has been given to geometric effects, specifically, the influence of long-chord-length crossings on the dose to the component of interest. It will be demonstrated, through an analysis of chord length distributions, that these long-chord-length crossings, despite their relative infrequent occurrence, are too important to be ignored and must be considered to completely assess an electronic component's susceptibility to SEEs. 
Studying the chord length distributions produced by random traversals relating to radiation hardness testing is not a novel concept. Previous work has considered both the differential [10-12] and integral [11] chord length distributions. What is unique, however, is the present consideration of the dose distributions for chord length crossings in addition to the commonly used frequency distribution.

\section{Methods}

The original driving force in the evolution into modern microdosimetry was the need to characterize the LET of the incident radiation as it is used somewhat as a marker of the radiation's damaging ability [1]. LET, commonly used in radiation hardness testing of microelectronics, is a macroscopic average of the energy lost by the incident radiation in the direction of traversal and classically carries units of $\mathrm{MeV} / \mathrm{cm}$ [13]. For use in radiation biology and due to the size of the target studied, these units were seen as less than sufficient so a factor of 10 is employed converting LET into units of $\mathrm{keV} / \mu \mathrm{m}$, where $10 \mathrm{MeV} / \mathrm{cm}=1 \mathrm{keV} / \mu \mathrm{m}$, now much more meaningful to the volumes of concern. This, however, does not solve the problem that LET is a one-dimensional concept that does not describe the radial extent of energy transfer $[14,15]$. At the macroscopic level, different incident particles may have the same LET at some point along their path but, due to the dependence of secondary delta-ray energy as a function of velocity, the local energy deposited may be very different due to energy escaping the volume of interest. This radial energy loss is a factor of both the incident ion velocity and the target geometry. Therefore, microscopic energy measurements are commonly presented in terms of the stochastic energy deposition or dose distributions in the volume and geometry relevant to the study [3].

The most common stochastic value used in microdosimetry measurements and simulations is lineal energy. The lineal energy $y$ is

$$
y=\frac{\epsilon}{\bar{l}}
$$

where $\epsilon$ is the energy imparted to the matter in a given volume by a single energy-deposition event and $\bar{l}$ is the mean chord length of the volume [3]. The mean chord length of a convex body is

$$
\bar{l}=\frac{4 V}{S},
$$

where $V$ is the volume and $S$ is the surface area [16].

Microdosimetry spectra [3] are commonly expressed in terms of the lineal energy $y$, its differential distribution $f(y)$, and the mean of this distribution $\bar{y}_{F}$ which is

$$
\bar{y}_{F}=\int_{0}^{\infty} y f(y) \mathrm{d} y .
$$

The dose distribution, reflecting the fact that higher lineal energies deposit a higher dose [17], is also often considered where

$$
d(y)=\frac{y f(y)}{\bar{y}_{F}}
$$

and the mean of this distribution, the dose mean lineal energy, is

$$
\bar{y}_{D}=\frac{1}{\bar{y}_{F}} \int_{0}^{\infty} y^{2} f(y) \mathrm{d} y,
$$

where $\bar{y}_{F}$ is the frequency mean lineal energy from (3). The frequency mean lineal energy $\bar{y}_{F}$ is the first moment of the $f(y)$ distribution and the dose mean lineal energy $\bar{y}_{D}$ is the ratio of the second and first moments of the $f(y)$ distribution [3].

For the case of radiation hardness testing, the methodology of $y$ can be applied to the chord length of a convex body. Thus, the frequency distribution of the chord length $f(l)$ has a mean value of

$$
\bar{l}_{F}=\int_{0}^{\infty} l f(l) \mathrm{d} l,
$$

where $l$ is the differential chord length. The frequency mean chord length $\bar{l}_{F}$ calculated by (6) is the numerical equivalent to the analytical value $\bar{l}$ calculated by (2). It must be kept in mind that this value is the frequency mean chord length and this equivalence between $\bar{l}_{F}$ and $\bar{l}$ only exists because of the isotropic nature of space radiation. This equivalence does not exist for nonisotropic sources.

Likewise, again applying the fact that longer chord lengths result in a higher energy imparted [17], the dose distribution is

$$
d(l)=\frac{l f(l)}{\bar{l}_{F}}
$$

with a mean value of

$$
\bar{l}_{D}=\frac{1}{\bar{l}_{F}} \int_{0}^{\infty} l^{2} f(l) \mathrm{d} l,
$$

where, again, $l$ is the differential chord length. These chord length mean values have the same relationship to the $f(l)$ distribution as the $y$ mean values did to the $f(y)$ distribution. The frequency mean chord length $\bar{l}_{F}$ is the first moment of the $f(l)$ distribution and the dose mean chord length $\bar{l}_{D}$ is the ratio of the second and first moments of the $f(l)$ distribution.

The value $\bar{l}_{D}$ is the dose mean chord length, that is, the chord length equal to the mean of the $d(l)$ distribution. It is these frequency and dose mean chord lengths, and perhaps more importantly the difference between the two, that are of interest in radiation hardness testing. As will be seen in the next section, these long-chord-length crossings contribute significantly to the total dose despite their relative infrequency.

A simple Monte Carlo algorithm, based on previous work [18] and utilizing a linear congruential random number generator [19], was implemented to produce the chord length frequency distributions for each geometry. These chord length distributions are equivalent to the path length distributions that would result from exposure to an isotropic source of charged particles. The novelty of this methodology 


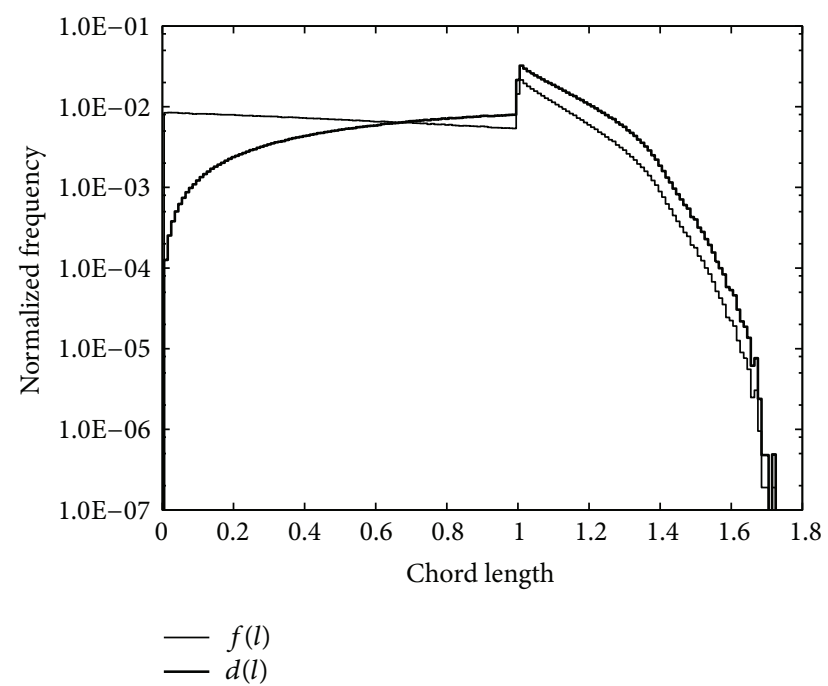

FIGURE 1: $f(l)$ and $d(l)$ distributions for a rectangular parallelepiped with relative dimensions $1 \times 1 \times 1$.

is contained within the analysis of the chord length distributions; any Monte Carlo algorithm that is capable of generating frequency distributions of chord length will suffice.

Starting with the code-produced frequency distribution $f(l)$, the frequency mean chord length $\bar{l}_{F}$ was calculated according to (6), the dose distribution $d(l)$ was produced according to (7), and the dose mean chord length $\bar{l}_{D}$ was calculated according to (8). This work is limited in scope to the rectangular parallelepiped (RPP) but the method is applicable to all convex bodies.

\section{Results and Discussion}

Frequency and dose distributions of chord length crossings for RPPs of four unique relative dimensions are presented. In each plot, the normal-line-weight curve represents the frequency distribution $f(l)$ and the heavy-line-weight curve represents the dose distribution $d(l)$.

Figure 1 shows the $f(l)$ and $d(l)$ distributions for a RPP with relative dimensions $1 \times 1 \times 1$, a cube. The peak at 1.0 corresponds to the dimension of the same value. Figures 2, 3 , and 4 show the $f(l)$ and $d(l)$ distributions for a noncubic RPP with relative dimensions $2.5 \times 1 \times 0.625,2 \times 2 \times$ 0.5 , and $4 \times 4 \times 0.4$, respectively. Likewise, the peaks in these distributions correspond to the dimensions of the same value. Each frequency distribution was produced by $1 \times 10^{7}$ iterations of the Monte Carlo algorithm [18]. A comparison of $\bar{l}_{F}$ and $\bar{l}_{D}$ values for each geometry appears in Table 1 along with the percent differences between the two.

Each unique geometry presented has the same $\bar{l}_{F}$ value despite having unique $f(l)$ distributions. In each case, the $f(l)$ distribution was weighted by $l$ to produce $d(l)$, as described in (7). It is these differences in the $f(l)$ distributions, as well as the $l$ weighting found in the $d(l)$ distributions, that lead to the significant differences in the $\bar{l}_{D}$ values despite each geometry having the same $\bar{l}_{F}$ value.

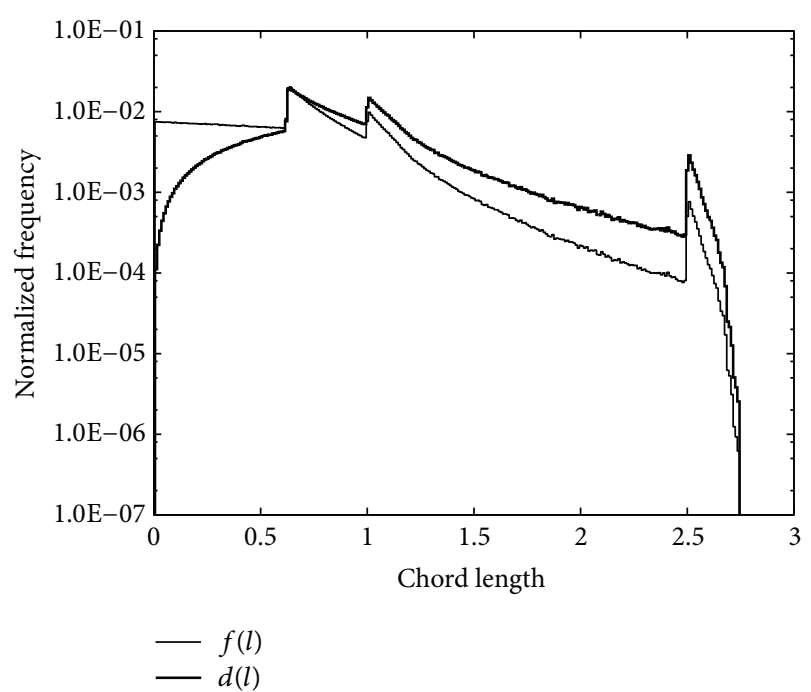

FIGURE 2: $f(l)$ and $d(l)$ distributions for a rectangular parallelepiped with relative dimensions $2.5 \times 1 \times 0.625$.

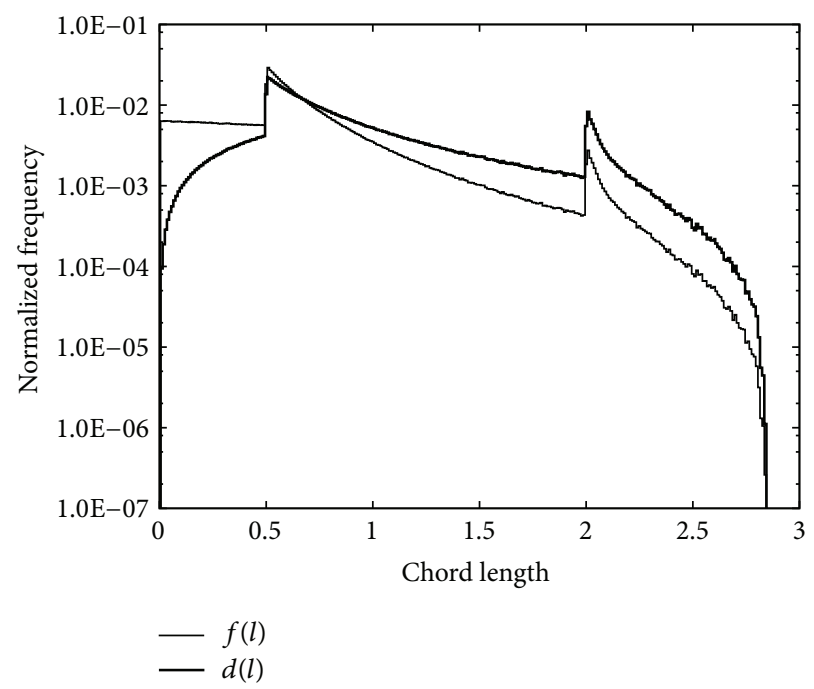

FIgURE 3: $f(l)$ and $d(l)$ distributions for a rectangular parallelepiped with relative dimensions $2 \times 2 \times 0.5$.

TABLE 1: $\bar{l}_{F}$ and $\bar{l}_{D}$ values for a selection of rectangular parallelepipeds of varying relative dimensions.

\begin{tabular}{lccc}
\hline Relative dimensions & $\bar{l}_{F}$ & $\bar{l}_{D}$ & \% difference \\
\hline $1 \times 1 \times 1$ & 0.667 & 0.897 & 34.5 \\
$2.5 \times 1 \times 0.625$ & 0.667 & 0.926 & 38.9 \\
$2 \times 2 \times 0.5$ & 0.667 & 0.964 & 44.6 \\
$4 \times 4 \times 0.4$ & 0.667 & 1.10 & 65.5 \\
\hline
\end{tabular}

While the long-chord-length crossings are quite rare, they represent a disproportionately high contribution to the total dose. This characteristic is made evident by large differences between the $\bar{l}_{F}$ and $\bar{l}_{D}$ values for each geometry presented in Table 1. Even for the least extreme case of the cube, $\bar{l}_{F}$ and $\bar{l}_{D}$ differ by $34.5 \%$. As the RPP becomes less cubic, the difference 


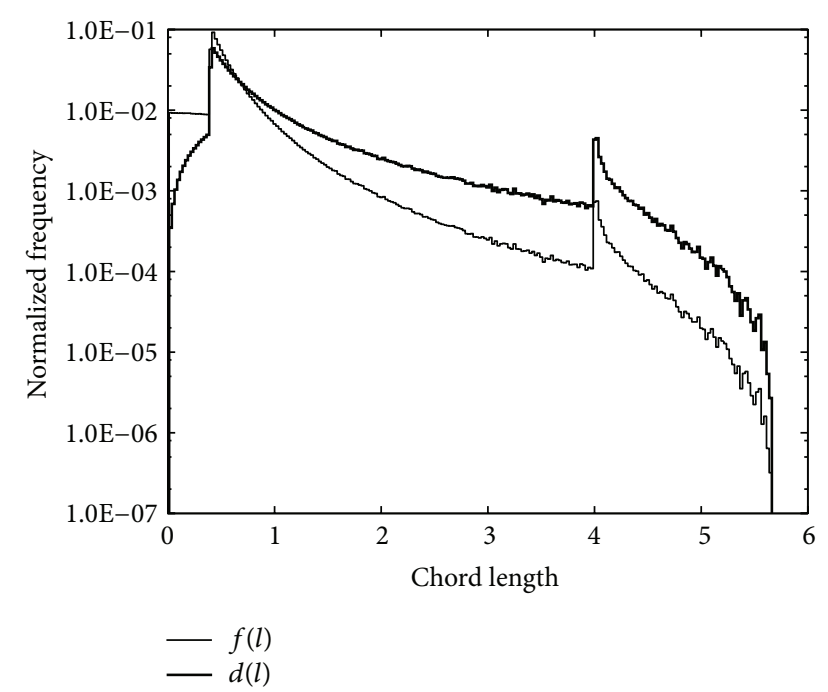

FIGURE 4: $f(l)$ and $d(l)$ distributions for a rectangular parallelepiped with relative dimensions $4 \times 4 \times 0.4$.

between $\bar{l}_{F}$ and $\bar{l}_{D}$ increases. For the most extreme case of the RPP with relative dimensions $4 \times 4 \times 0.4, \bar{l}_{F}$ and $\bar{l}_{D}$ differ by $65.5 \%$.

A quick comparison to previous work confirms the validity of the dose mean chord length methodology presented in (8). Since $\bar{l}_{D}$ is the ratio of the second and first moments of the $f(l)$ distribution, it would be expected that the product of $\bar{l}_{F}$ and $\bar{l}_{D}$ would be equal to the second moment of the $f(l)$ distribution. Simple arithmetic applied to the RPP of relative dimensions $1 \times 1 \times 1$, a unit cube, gives the second moment of the $f(l)$ distribution as

$$
\bar{l}_{F} \times \bar{l}_{D}=0.667 \times 0.897=0.598
$$

which matches the value of 0.5978 given by Coleman as the second moment of the distribution of the random path lengths through a unit cube [20].

\section{Conclusion}

Frequency and dose distributions of chord length crossings were presented for RPPs of varying relative dimensions, each with the same frequency mean chord length. The frequency mean chord length $\bar{l}_{F}$ and dose mean chord length $\bar{l}_{D}$ were presented for each unique geometry. In every case, $\bar{l}_{D}$ was greater than $\bar{l}_{F}$. Even for the least extreme case of the cube, $\bar{l}_{F}$ and $\bar{l}_{D}$ differ by more than $30 \%$. The significant differences presented between $\bar{l}_{F}$ and $\bar{l}_{D}$ illustrate the importance of considering long-chord-length crossings in radiation hardness testing despite their relatively low probability of occurrence.

\section{Conflict of Interests}

The authors declare that there is no conflict of interests regarding the publication of this paper.

\section{Acknowledgments}

This work was supported by the U.S. Nuclear Regulatory Commission, Faculty Development Program, Grant no. NRC-38-10-923, Contract no. C11-00494. The authors would like to thank Z. Kulage for help with troubleshooting the code.

\section{References}

[1] A. M. Kellerer, "Microdosimetry: reflections on Harald Rossi," Radiation Protection Dosimetry, vol. 99, no. 1-4, pp. 17-22, 2002.

[2] J. Booz, L. Braby, J. Coyne et al., "Microdosimetry," Tech. Rep. 36, International Commission on Radiation Units and Measurements, Bethesda, MD, USA, 1983.

[3] H. H. Rossi and M. Zaider, Microdosimetry and Its Applications, Springer, New York, NY, USA, 1996.

[4] K. P. Rodbell, D. F. Heidel, H. H. K. Tang, M. S. Gordon, P. Oldiges, and C. L. Murray, "Low-energy proton-induced singleevent-upsets in $65 \mathrm{~nm}$ node, silicon-on-insulator, latches and memory cells," IEEE Transactions on Nuclear Science, vol. 54, no. 6, pp. 2474-2479, 2007.

[5] J. A. Pellish, M. A. Xapsos, K. A. Label et al., "Heavy ion testing at the galactic cosmic ray energy peak," in Proceedings of the 10th European Conference on Radiation and Its Effects on Components and Systems (RADECS '09), pp. 559-562, September 2009.

[6] D. F. Heidel, K. P. Rodbell, P. Oldiges et al., "Single-event-upset critical charge measurements and modeling of $65 \mathrm{~nm}$ siliconon-insulator latches and memory cells," IEEE Transactions on Nuclear Science, vol. 53, no. 6, pp. 3512-3517, 2006.

[7] J. A. Pellish, M. A. Xapsos, K. A. Label et al., "Heavy ion testing with iron at $1 \mathrm{GeV} / \mathrm{amu}$," IEEE Transactions on Nuclear Science, vol. 57, no. 5, pp. 2948-2954, 2010.

[8] V. Ferlet-Cavrois, J. R. Schwank, S. Liu et al., "Influence of beam conditions and energy for SEE testing," IEEE Transactions on Nuclear Science, vol. 59, no. 4, pp. 1149-1160, 2012.

[9] J. R. Schwank, M. R. Shaneyfelt, V. Ferlet-Cavrois et al., "Hardness assurance testing for proton direct ionization effects," IEEE Transactions on Nuclear Science, vol. 59, no. 4, pp. 11971202, 2012.

[10] S. R. Messenger, E. A. Burke, G. P. Summers, and R. J. Walters, "Limits to the application of NIEL for damage correlation," IEEE Transactions on Nuclear Science, vol. 51, no. 6, pp. 3201-3206, 2004.

[11] G. I. Zebrev, I. O. Ishutin, R. G. Useinov, and V. S. Anashin, "Methodology of soft error rate computation in modern microelectronics," IEEE Transactions on Nuclear Science, vol. 57, no. 6, pp. 3725-3733, 2010.

[12] J. Barak, "Analytical microdosimetry model for proton-induced SEU in modern devices," IEEE Transactions on Nuclear Science, vol. 48, no. 6, pp. 1937-1945, 2001.

[13] F. H. Attix, Introduction To Radiological Physics and Radiation Dosimetry, John Wiley \& Sons, Weinheim, Germany, 2004.

[14] A. Chatterjee and H. J. Schaefer, "Microdosimetric structure of heavy ion tracks in tissue," Radiation and Environmental Biophysics, vol. 13, no. 3, pp. 215-227, 1976.

[15] E. J. Kobetich and R. Katz, "Energy deposition by electron beams and $\delta$ rays," Physical Review, vol. 170, no. 2, pp. 391-396, 1968.

[16] A. Cauchy, "Mmoire sur la rectification des courbes et la quadrature des surfaces courbes," in Oeuvres Compltes, pp. 167177, Gauthier, Paris, France, 1908. 
[17] P. D. Bradley, A. B. Rosenfeld, and M. Zaider, "Solid state microdosimetry," Nuclear Instruments and Methods in Physics Research B, vol. 184, no. 1-2, pp. 135-157, 2001.

[18] D. A. Rajon and W. E. Bolch, "Interactions with 3D isotropic and homogeneous radiation fields: a Monte Carlo simulation algorithm," Computer Methods and Programs in Biomedicine, vol. 70, no. 2, pp. 167-177, 2003.

[19] F. B. Brown and Y. Nagaya, "The MCNP5 random number generator," Transactions of the American Nuclear Society, vol. 87, pp. 230-232, 2002.

[20] R. Coleman, "Random paths through rectangles and cubes," Metallography, vol. 6, no. 2, pp. 103-114, 1973. 


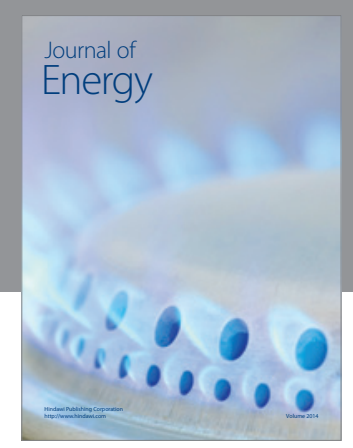

Journal of

Industrial Engineering
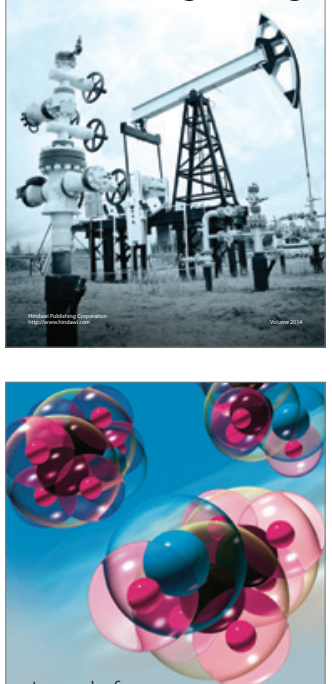

Fuels
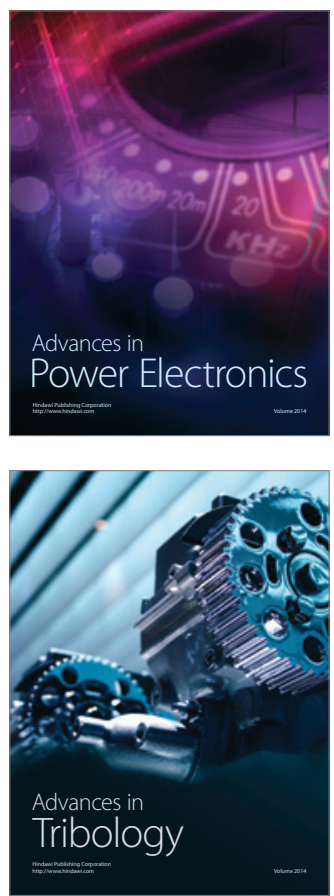

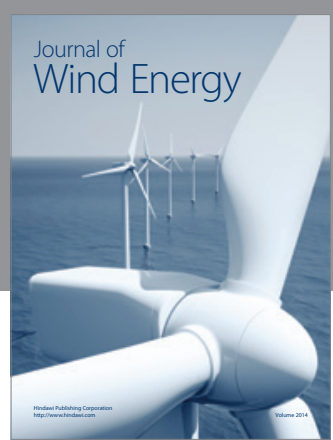

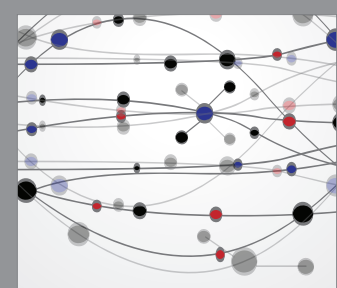

The Scientific World Journal

Submit your manuscripts at http://www.hindawi.com

Journal of

Structures
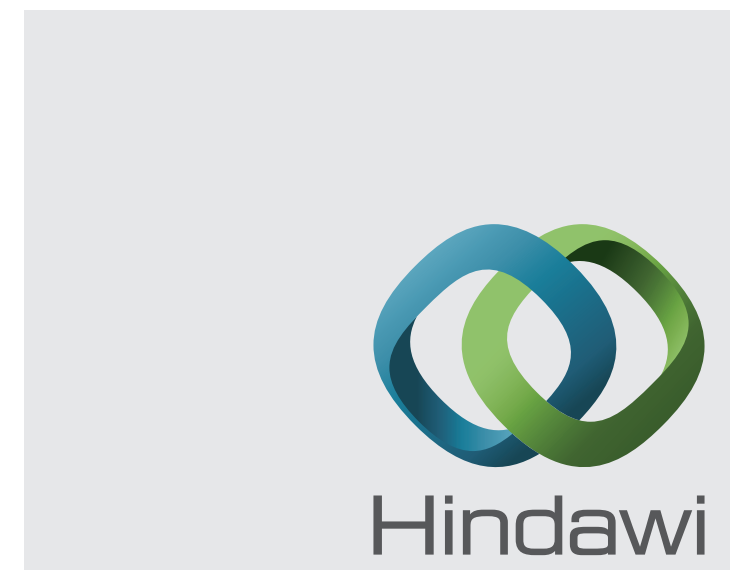

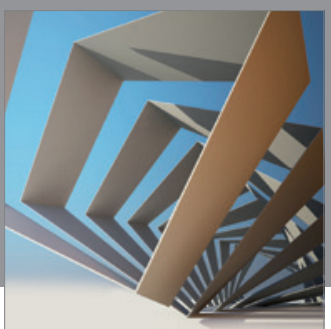

Rotating

Machinery
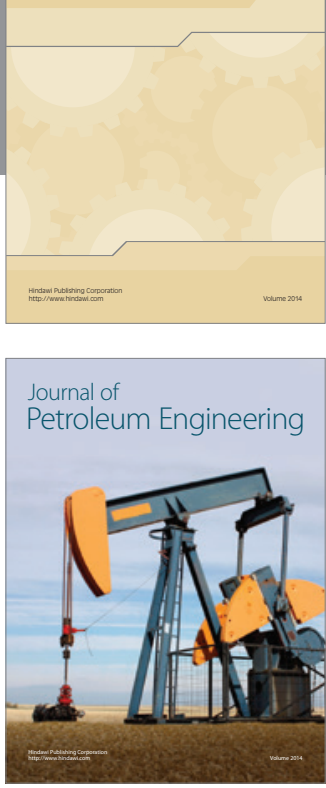

Journal of

Solar Energy
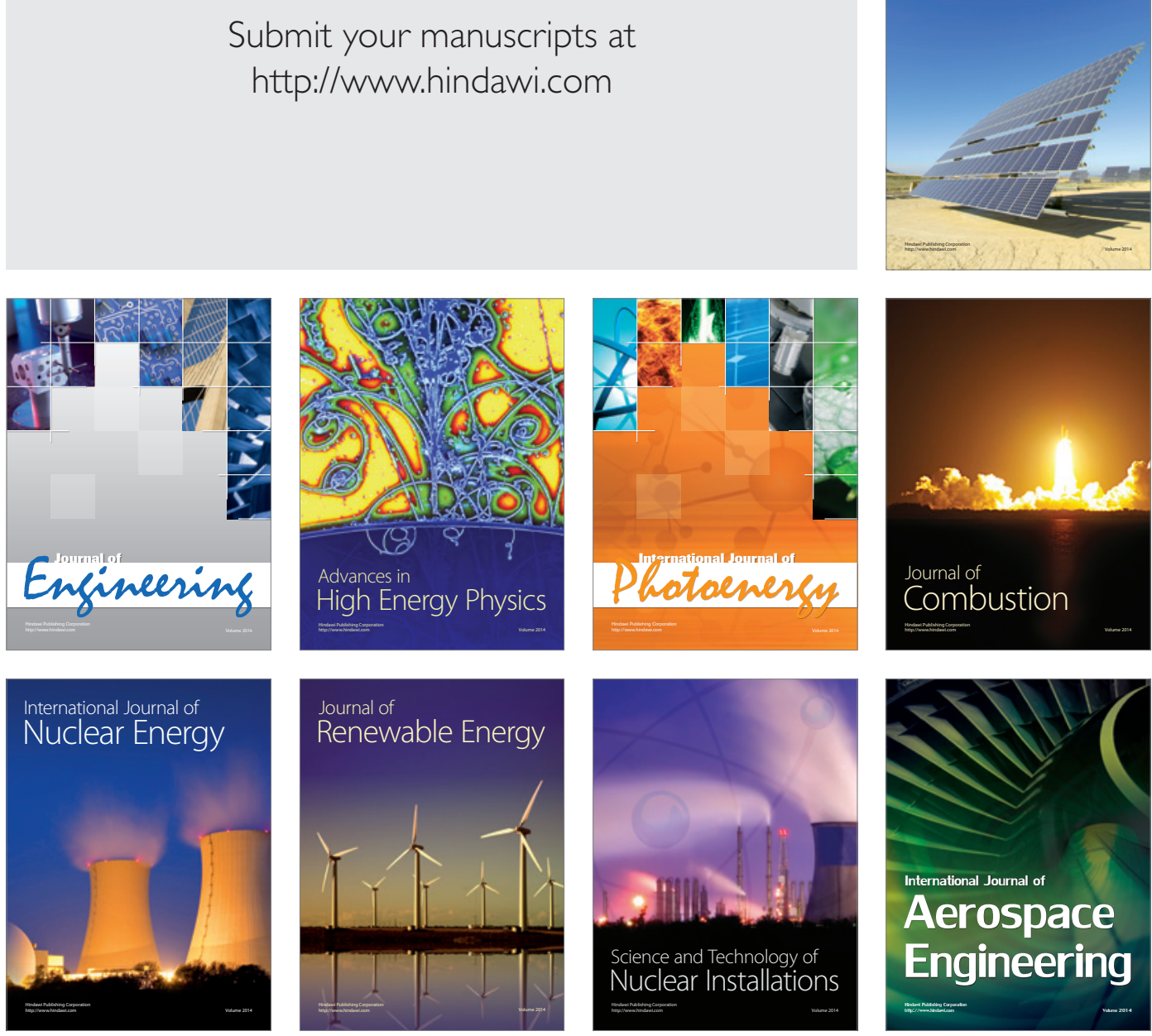